\title{
Design and Analysis of an Unmanned Aerial Vehicle Capable of Carrying the Camera
}

\author{
Mohammadmahdi Khamsi
}

Department of Mechanics, University of Guilan, Iran

Copyright $@ 2019$ by authors, all rights reserved. Authors agree that this article remains permanently open access under the terms of the Creative Commons Attribution License 4.0 International License

\begin{abstract}
Today Unmanned aerial vehicles (UAV) have found widespread use and may have been using them to capture various events or take photographs of operational areas; but the fact is that UAVs or quadcopters, which are some of these flights, are still at the beginning of their journey and can be future applications will be more widely used. Given the geographical situation and the current situation in the world, the country needs to step up with the world in order to strengthen the defense forces. One of the tools that have been used in recent decades to other tools due to their characteristics and their unique features, including the absence of human casualties, the study and more precise missions, and ... are of interest to many countries, including our country, are unmanned aerial vehicles. Many researchers have been carried out on these devices today. In the same way, our researchers in our country have done a lot of researches on this device in recent years and tried to make these birds with the least cost and with the highest efficiency. This paper also attempts to analyze the flights with the ability to carry the camera. At first, its components, including wings, body, tail, and landing gear, are optimally designed and then aerodynamically and instrumentally analyzed, so that this research used to build these flights species in the future.
\end{abstract}

Keywords Unmanned Aerial Vehicles, Quad Copters, Camera

\section{Introduction}

UAVs have reached significant attention in recent years due to their suitability for a broad variety of civil, military, and societal missions. Development of UAVs using the properties of multi-rotor UAV is focused [1].

The UAV system is equipped with various intelligent sensors such as barometer, Inertial Navigation System (INS), Global Positioning System (GPS), flight control, navigation control, sonar, and infrared and Electronic Speed Controller (ESC) [2]. This study attempted to the development of UAVs for constructed micro UAV [3, 4, 5].

Unmanned aerial vehicles (UAVs) fill a gap between satellites and aircraft when a stationary monitoring platform is needed for relatively long-term observation of an area. UAVs have advanced designs to carry small payloads and integrated flight control systems, giving them semiautonomous or fully autonomous flight capabilities [6].

In recent years rapid advances in the quality, availability, and range of sensors in commercial unmanned aerial vehicles (UAVs) have led to their widespread use in the fields of ecology and conservation [7, 8, 9].

One of the defensive and research tools used today in the world is unmanned aerial vehicles due to its unique features, such as: no human casualties, more precise missions, less overall cost, and more ... more countries in the world such as our country is also trying to build and design these aerial vehicles [10,11, 12, 13, 14].

A further advantage of UAVs that is seldom raised is that the aerial photography based approach provides a bank of images from which individual species can be independently counted, verified and archived for future analysis or audit. This creates a transparent census technique that increases the usability and cost-effectiveness of information gathered if images are made available to other researchers $[15,16]$.

Yayla et al. [10] performed theoretical analysis to investigate the performance characteristics such as rate-of-climb, turn radius, and maximum velocity of an amphibious UAV.

Sutheerakul et al (2017) focus on applying UAV to monitor pedestrian traffic flows and using data collected from UAVs to manage pedestrian demand and supply. UAV is capable of carrying a video camera to record high-quality images and real-time videos, and a global positioning system to transmit spatial and temporal data to the ground. The efforts are made for two thrusts. The results showed that UAV can be an alternative viable technology in monitoring pedestrian traffic characteristics in outdoor pedestrian zones. Data collected from UAV 
technology can be used to develop traffic demand and supply management plans in a more efficient and cost-effective way than conventional data collection techniques [18].

McEvoy et al (2016) assessed the level of disturbance that a range of UAV shapes and sizes had on free-living, non-breeding waterfowl surveyed in two sites in eastern Australia between March and May 2015, as well as the capability of airborne digital imaging systems to provide adequate resolution for unambiguous species identification of these taxa. The results show that with proper planning of take-off and landing sites, flight paths and careful UAV model selection, UAVs can provide an excellent tool for accurately surveying wild waterfowl populations and provide archival data with fewer logistical issues than traditional methods such as manned aerial surveys [19].

Al Nuaimi et al (2018) conduct a feasibility study for developing a potential UAV based persistent surveillance system with tethered power supply. The majority of the unmanned aerial vehicles are able to carry optics payloads, allowing them to take aerial images from strategic viewpoints. The constrained onboard processing power in addition to the strict limit in the flying time of UAV are amongst the serious challenges that have to be overcome to enable a cost-effective persistent surveillance based on UAV platforms [20].

Franco et al (2017 introduces a low-cost embedded system design and implementation for a two-axis camera platform control used in UAVs. The main goal is to have a gimbal able to stabilize a camera, with respect to UAV attitude, using permanent magnet synchronous motors as actuators. Their design also uses an inertial measurement unit as a sensor and a proportional-integral-derivative controller. Simulated and experimental results show that the developed embedded system was able to successfully respond to a simulated UAV flight envelope, reacting in real-time and minimizing those disturbances [21].

Torres-Sánchez et al (2013) describe the technical specifications and configuration of a UAV used to capture remote images for early season site-specific weed management (ESSWM). These results suggest that an agreement among spectral and spatial resolutions is needed to optimize the flight mission according to every agronomical objective as affected by the size of the smaller object to be discriminated [22].

\section{Dynamic Model of UAV}

To be able to design a suitable controller for a system, having sufficient knowledge of the dynamics of the system is very important. In this section, the dynamical equations governing the system are presented. The motion equations in three directions (length, height, and width) are (Fig. 1) [23].

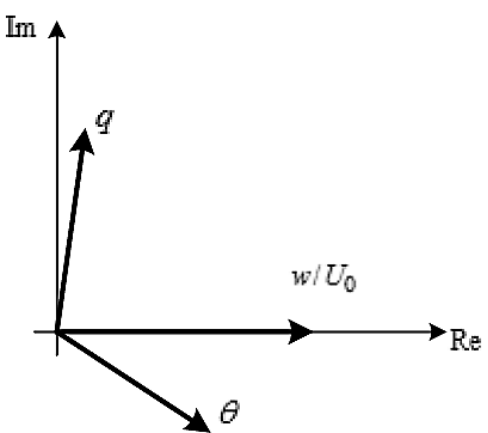

Figure 1. Phasor diagram in longitudinal for long flight mode

$$
\begin{gathered}
\left(\frac{m u}{s q} s-C_{X_{u}}\right)^{\prime} u(s)-C_{X_{a}}{ }^{\prime} a(s)-C_{w}(\cos \Theta) \theta(s)=0 \\
-C_{Z_{u}}{ }^{\prime} u(s)\left[\left(\frac{m u}{s q}-\frac{c \cdot C_{Z \dot{a}}}{2 u}\right) s-C_{Z \dot{a}}\right] ' a(s) \\
+\left[\left[\left(-\frac{m u}{S q}-\frac{c \cdot C_{Z \dot{a}}}{2 u}\right) s-C_{w}(\sin \Theta)\right] \theta(s)\right] \\
\left(-\frac{c \cdot C_{Z \dot{a}}}{2 u} s-C_{M_{u}}{ }^{\prime}\right) a(s)+\left(\frac{I_{y}}{s q c} s^{2}-\frac{c \cdot C_{Z \dot{a}}}{2 u} s\right) \theta(s)=0
\end{gathered}
$$

Where ' $u$ velocity changes in the longitudinal direction of flight and ' $a$ are angle change in the motion in the flight path, $\Theta$ is the pitch angle, $\theta$ is the pitch angle changes relative to the steady state and therefore ' $u=u / U_{0}$ and ' $a=\frac{w}{U_{0}}$ where $u$ is the velocity changes in the longitudinal direction, $\mathrm{w}$ is the speed deviation in the altitude path and $U$ is the longitudinal velocity of the aircraft in a steady state. Also, other required coefficients are presented in Tables 1 and 2.

Table 1. The coefficients of a UAV [23]

\begin{tabular}{|c|c|}
\hline Coefficient & Value \\
\hline $\mathrm{m}$ & $6[\mathrm{Kg}]$ \\
\hline$U_{0}$ & $10[\mathrm{~m} / \mathrm{sec}]$ \\
\hline $\mathrm{g}$ & $8.908\left[\mathrm{~m} / \mathrm{sec}^{2}\right]$ \\
\hline $\mathrm{S}$ & $0.3890\left[\mathrm{~m}^{2}\right]$ \\
\hline$S_{\text {vertical tail }}$ & $0.184\left[\mathrm{~m}^{2}\right]$ \\
\hline$\rho$ & $2.0147\left[\mathrm{Kg} / \mathrm{m}^{3}\right]$ \\
\hline $\mathrm{I}_{y y}$ & $0.1587\left[\mathrm{~m}^{4}\right]$ \\
\hline $\mathrm{L}_{t / c}$ & $0.415[\mathrm{~m}]$ \\
\hline
\end{tabular}

Table 2. Stable inputs and converters of UAV [23]

\begin{tabular}{|c|c|}
\hline Coefficient/ Value & Coefficient/ Value \\
\hline $\mathrm{L}_{t / c}=1$ & $\mathbf{C}_{\boldsymbol{M \& e}}=-\mathbf{0 . 8 7}$ \\
\hline $\mathrm{C}_{L}=1.6578$ & $\mathbf{C}_{\boldsymbol{X} \& e}=\mathbf{0}$ \\
\hline $\mathrm{C}_{D}=0.0245$ & $\mathbf{C}_{\boldsymbol{M q}}=-\mathbf{2 . 8 7}$ \\
\hline $\mathrm{C}_{W}=-1.8451$ & $\mathbf{C}_{\boldsymbol{M a}}=-\mathbf{0 . 0 4 5 7}$ \\
\hline $\mathrm{C}_{Z \& e}=-0.89$ & $\mathbf{C}_{\boldsymbol{M \boldsymbol { a } ^ { \prime }}}=-\mathbf{0 . 0 4 5 7}$ \\
\hline $\mathrm{C}_{Z u}=-2.2358$ & $\mathbf{C}_{\boldsymbol{Z q}}=-\mathbf{2 . 4 5 8 7}$ \\
\hline $\mathrm{C}_{x u}=-0.1245$ & $\mathbf{C}_{\boldsymbol{Z a}}=-\mathbf{1 . 4 5 8 7}$ \\
\hline $\mathrm{C}_{x a}=2.2145$ & $\mathbf{C}_{\boldsymbol{Z a}}=-\mathbf{1 . 6 4 5 8}$ \\
\hline
\end{tabular}


Since in this method, the pitch angle changes than $\delta_{e}$ considered, then conversion function $\theta / \delta_{e}$ is (Fig. 2):

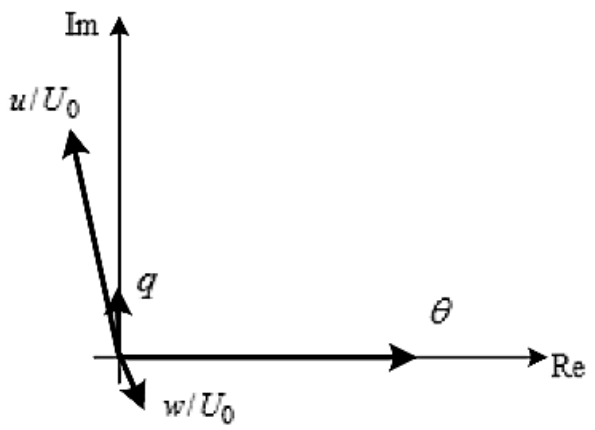

Figure 2. Phasor diagram in longitudinal for short flight mode

$\frac{\theta(s)}{\delta_{e}(s)}=\frac{1.423 s^{2}+0.134 s+1.836}{0.02424 s^{4}+0.06838 s^{3}+0.1 s^{2}+0.0859 s+0.08536}$

Also, system coefficients in linear flight mode are shown in Table 3.

Table 3. The values of UAV coefficients in the longitudinal flight path [23]

\begin{tabular}{|c|c|}
\hline Short flight mode & Long flight mode \\
\hline$\xi_{p m}=0.1015$ & $\xi_{\text {sm }}=\mathbf{0 . 4 5 1}$ \\
\hline$\omega_{p m}=1.1245 \mathrm{rad} / \mathrm{sec}$ & $\boldsymbol{\omega}_{s m}=\mathbf{2 . 0 0 1 2} \mathbf{~ a d} / \mathbf{s e c}$ \\
\hline $\mathrm{T}_{p m}=52.1025 \mathrm{sec}$ & $\mathbf{T}_{s m}=\mathbf{1 . 0 3 5 2} \mathbf{~ s e c}$ \\
\hline
\end{tabular}

\section{Initial Sample}

In a number of previous methods, variable features that are sensitive to scale and size are used.

However, in view of the characteristics of UAV which have wing and front section in accordance with Fig. 3, a model can be developed based on the characteristics of the above-mentioned problems to solve the problems in the previous methods .Many powerful methods, such as the simulation model, cannot be used in these sensitive applications because of the high complexity of time . Before we found the object, we were able to solve this problem using the technique of analysis of the main components trained by UAV models.

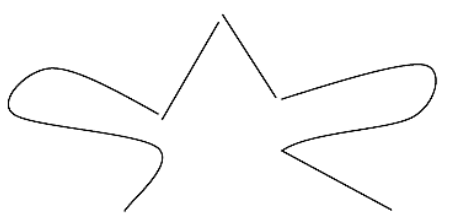

Figure 3. Different arrangement of the front of the UAV

\subsection{Weight Estimation and Sensitivity Analysis}

According to the specifications of the UAVs and based on their application, at the beginning of the design of the weight of the load (Wpayload) that is $3 \mathrm{~kg}$ and the weight take-off ("WTO") is considered to be $17 \mathrm{~kg}$. Therefore, for this UAV, the weight of the fuel (WF) is $2.32 \mathrm{~kg}$ and weight of the empty (WE) $12.5 \mathrm{~kg}$ were determined; empty weight of the UAV was equal to [24]:

$$
\begin{gathered}
W_{E}=0.3459 \times W_{T O}^{1.251} \\
W_{T O}=17^{\mathrm{kg}} \rightarrow W_{E}=10.89^{\mathrm{kg}}
\end{gathered}
$$

But by comparing this value, with the empty weight obtained from the fuel ratio method it was determined that the initial assume of the take-off weight and the weight of the load was not suitable. Therefore, using mentioned method the calculation steps were repeated again, so that the result was obtained with a percentage difference of $5 \%$ relative to the empty weight [24]:

$$
\begin{gathered}
W_{T O}=16.1 \mathrm{~kg}, W_{p}=3 \mathrm{~kg} \\
W_{F}=2.20 \mathrm{~kg}, W_{E}=9.88 \mathrm{~kg} \\
W_{T O}=15.6 \mathrm{~kg}, W_{p}=2.5 \mathrm{~kg} \\
W_{F}=2.12 \mathrm{~kg}, W_{E}=9.87 \mathrm{~kg}
\end{gathered}
$$

According to the above results, the takeoff weight of the UAV is equal to $16.1 \mathrm{~kg}$. The amount of empty weight (due to available technologies and construction problems) should be considered slightly higher than the calculated value. Therefore, the empty weight of UAV was set at $11.31 \mathrm{~kg}$. According to the following equation, which indicates the fuel weight of the UAVs in terms of take-off weight, some of the load weight should be reduced and added to the calculated fuel weight. So the fuel weight of UAV would be $2.61 \mathrm{~kg}$ [24].

$$
\begin{aligned}
W_{E} & =0.2134 \times W_{T O}^{1.142} \\
W_{T O} & =16.57^{\mathrm{kg}} \rightarrow W_{F}=2.54^{\mathrm{kg}}
\end{aligned}
$$

Since the final calculations have added to the empty weight and fuel weight of the UAV, the load weight should be reduced to the same extent. As a result, the load weight of the UAV will be $2 \mathrm{~kg}$ which is in our design a reasonable amount.

\subsection{The Process of Estimating the Wing Area and Engine Power}

In order to estimate the wing area and maneuver power, all critical flight phases should be considered and according to the constraints in each design, phase performed. For this purpose, the matching diagram method has been used. According to this method, the critical point of the design, the location of the intersection of the outbursts and take-off lines was determined, which required the maximum engine power and minimum wing area. So we will have [25]:

$$
\begin{aligned}
& W / S=212.8 \mathrm{~N} / \mathrm{m}^{2} \\
& P / W=16^{\mathrm{W}} / \mathrm{N}
\end{aligned}
$$


Given the values obtained, and considering the take-off weight of $16.1 \mathrm{~kg}$, the required wing area and engine power requirements are as follows [25]

$$
\begin{gathered}
S=0.6 \mathrm{~m}^{2} \\
P=2504 W=3.2 h p
\end{gathered}
$$

\subsection{Engine Configuration}

Table 4. The chosen engine has the following specifications

\begin{tabular}{|l|l|}
\hline Engine Configuration \\
\hline Engine type & Single piston two stroke \\
\hline Horsepower & $3.7 \mathrm{HP}$ @ 12600 RPM \\
\hline RPM & 2000 to 12600 RPM \\
\hline Weight-Engine Alone & $1.85 \mathrm{lbs}(897 \mathrm{~g})$ \\
\hline Weight with muffler & $2.45 \mathrm{lbs}(1077 \mathrm{~g})$ \\
\hline Overall length & $112.5 \mathrm{~mm}(4.63 ")$ \\
\hline Width & $46 \mathrm{~mm}(1.93 ")$ \\
\hline Height & $112.5 \mathrm{~mm}(4.63 ")$ \\
\hline Fuel Type & Nitro \\
\hline Price & $\$ 3000$ \\
\hline
\end{tabular}

One of the initial design steps in determining engine is to select the correct engine from several standard engines used in similar UAVs. Some of the parameters in this area are: maximum power required with the maximum required motive force, maximum of UAV flight, installed engine weight, specific fuel consumption, operating and maintenance costs of the engine, engine price, volume of interrupted noise, change motive force change with fluctuation conditions (humidity, temperature, density, height, and speed), and velocity adjustment limited by examining these parameters, it was found that the Q400M piston engine- a Canadian company was designed with a power about 3000 watts, which has a volume is equal to 410 cubic meters and locusts with $0.25 * 0.45 \mathrm{~m}$ can be achieved with good yield and distance of about 848 radians per second to flow out. The propeller type also has two blades at a constant speed. The single-cylinder engine of the UAV has a maximum diameter of about 0.022 meters and is placed at the end of the body as a propulsion motor. It also weighs 1.85 kilograms.

\subsection{Body Design}

The main parts of the body are: locations and loading compartment of the load and additional loads, location of the engine (end part of the body), location of equipment for takeoff and landing, location of electrical and hydraulic systems (nose section), plates and clamps related to the body wing, body canvas, engine-body, doors for fixing the systems and placing the cargo in the body, therefore, in light of the above conditions, and taking a look at other UAVs, the various parameters of the body are specified: the body weight ratio (the ratio of the length of the body to its largest diameter) as one of the important quantities is determined by this principle. This ratio usually varies between 3 and 14, and varies between 5 and 7 in planes. Therefore, according to the features of the design, the number of 3.5 has been selected. The maximum diameter of the UAV, according to the diameter of the engine and the load, is 0.2 meters, so the body will have a length of 1.06 meters. In the case of UAV widths, also with UAVs surveys, generally, it is generally known that the maximum width of UAVs is approximately 0.001 meters smaller than the maximum UAV height. Therefore, according to the engine's diameter and appearance of the section, the value of $0.02 \mathrm{~m}$ is chosen as the width of the body. The ratio of the length of the wing to the length of the body, as well as a roughly constant value of about $0.61 \mathrm{~m}$, is similar in the UAV. Therefore, the wings will be equal to 0.65 meters from the nose.

With regard to the numbers obtained and the strength expected from the tail in its specific design, the ratio of the total length of the UAV to the length of the body will be equal to 1.8 . As the length of the body is $1.06 \mathrm{~m}$, the total length of the body of the UAV from the nose to the tail is 1.9 meter.

\subsection{Dimensional Analysis of Tools for Increasing Lift Force}

In this section, coefficients are obtained for UAV in different flight modes. Here, the coefficient for the maximum for a uniform flight, as well as for lift force, which sets the highest lift coefficient for each flight, should be determined. Statistically, the coefficient for a UAV on a uniform flight is usually considered to be 1.6. Calculations also show that this coefficient is a reasonable coefficient [25].

$$
\begin{gathered}
W=\frac{1}{2} \times \rho \times V^{2} \times S \times C_{1} \\
V=14.12 \mathrm{~m} / \mathrm{s}
\end{gathered}
$$

Because the speed attained above is at the same time close to the speed of UAV lay-offs, and given that the uniform flight is at a speed of $22 \mathrm{~m} / \mathrm{s}$, so we will not be able to get close to the usual speed. Secondly, the selected hood satisfies this feature. It is stated [25]:

$$
\begin{aligned}
& C_{L_{\max \text { clean }}}^{\text {wing }}=1.1 \text { or } 1.05 C_{L_{\max } \text { clean }}^{A / C} \\
& C_{L_{\max \text { clean }}}^{\text {wing }}=1.05 \times 1.7=1.65
\end{aligned}
$$


Notice that $L_{h} / \bar{C}$ in the UAV is above 5 , or in other words is "long coupled". So, high equity ratio in this calculation is equal to 1.05 . Also, since the wing of this plane is rectangular and not narrowed, it also does not have a rollback angle, therefore [25]

$$
C_{L_{\text {max clean }}^{\text {Airfoil }}}^{\text {Ale }}=C_{L_{\text {max clean }}^{\text {wing }}}^{\text {wat }}=1.65
$$

According to these calculations, our UAV needs to have a hood factor of 1.68. By reviewing existing hoods, the Wortman FX76MIP hood achieves the highest coefficient for their production of 1.7. So the use of this hood is confirmed. The tail-design of the UAV uses a V-shaped tail.

Due to the fact that the engine is located on the back and the bottom of the aircraft body and is of a type of propeller, the air flow is flowing with high velocity on the tail section.

Therefore, we will have high aerodynamic pressure (Q). For this reason, $\mathrm{S}$ can be considered less than the tail area.

As a result, the weight of the tail structure is reduced, but the amount of vibration of the UAV will increase anyway. In this UAV, the distance between two canvases can be considered at a distance of 0.4-1.6 m, but due to issues such as tail size, structural weight, locomotive diameter and its safe distance, the distance between the two canvases on each side of the grasshopper was 0.1 meters and finally the gap between the canvases was 0.7 meters.

The length of canvas from the end of the grasshopper is also 0.8 meters. The horizontal and vertical tail area is also an important parameter in design. In different UAVs, the horizontal tail to the wing level ratio $\left(S_{h} / S\right)$ in the range of $0.12-0.21 \mathrm{~m}$, as well as the vertical tail to wing ratio $\left(S_{v} / S_{S}\right)$ in the range from 0.04 to $0.102 \mathrm{~m}$ is variable. Therefore, since the value of $\mathrm{S}$ cannot be considered too large, the number of 0.15 for the horizontal tail and 0.50 for the vertical tail are taken into account [25].

$$
\begin{aligned}
& \frac{S_{h}}{s}=0.15 \rightarrow S_{v}=0.102 \mathrm{~m}^{2} \\
& \frac{S_{v}}{s}=0.05 \rightarrow S_{v}=0.036 \mathrm{~m}^{2}
\end{aligned}
$$

Also, since the torque arm of the tail is less than other $\mathrm{UAV}$, and, on the other hand, the area of the tail is somewhat average, therefore, a flap with a width of $0.6 \mathrm{~m}$ the wing chord is used.

\subsection{Find the Coordinates of the Base of the Mass Center}

The general components of this aircraft are: a body including shell and structure (ramps and Langdon of the body, etc.), engine, fuel, avionics systems, automatic communication systems and pilot, cargo, wing, the tail, canvas connect tail to wings. In these components, the empty the weight of the aircraft include: body weight, structure, etc., engine weight, weight of equipment. The main thing in estimating the weight of the body is that the estimates are relatively modest and that the weight of the structure in the manufacturing process can be weighed less than the estimated weight.

So the weight saved can be added to the electronic equipment or the cargo weight of the aircraft. After finding the location of the center of mass, according to the fuel consumption, as well as the composition of the aircraft, the center of mass changes are calculated.

\subsection{Landing Gear}

The UAV has a tricycle landing gear. The position of the landing gear is the nose $0.16 \mathrm{~m}$ from the tip of the body. The longitudinal distance of the wheels of the landing gear is also determined by considering the safety margin of 0.55 meters and the transverse distance of the landing gear 0.7 meters considered. The height of the landing gears is also estimated at 0.25 meters.

\subsection{Sustainability}

The purpose of this section is to examine the longitudinal stability as well as the transverse aspect of the intrusion of the UAV. This section has a significant role in measuring and verifying the data in the previous steps. Because the UAV does not have inherent stability, all or parts of the design in the previous steps should be corrected. An examination of inherent longitudinal stability for inherent longitudinal stability must be [25]:

$$
\bar{X}_{a c A / C}-\bar{X}_{c g} g_{\bar{C}} \geq 0.1
$$

So

$$
\bar{X}_{a c A / C}-\bar{X}_{c g} g_{\bar{C}}=4.05-3.6=0.56>0.1
$$

The above value, although it is large, but meets the needs of the UAV to provide inherent sustainability, indicating that the UAV has a very high ratio for wing, as well as wing sections, and a fairly large horizontal tail with a remarkable distance from the center of the mass, it has very low maneuver power and is very stable. To reduce this stability, it is possible to near the tail to the body, reduce its area, or reduce the aspect ratio of the wing. But overall, the amount obtained is an acceptable amount.

Inherent transverse stability analysis of this section involves examining the changes $C_{n}$ in the UAV in terms of lateral angle changes [25].

$$
\begin{gathered}
C n_{\beta A / C}=C n_{\beta F}+C n_{\beta W}+C n_{\beta v r} \\
C n_{\beta A / C}=-0.079+0+0.112=0.054
\end{gathered}
$$

It can be seen that the UAV has inherent stability along the transverse side. But usually, in assessing the stability of UAVs, the ability of the UAV moving the vertical rudder to control it when the engine is lost is also measured. Due to the single engine of the above-mentioned UAV and the lack of a passenger, the necessity of this will be eliminated. 


\subsection{Fuel System Design}

In the first step, the design of the UAV's fuel system, due to the wing low thickness and the amount of fuel required, is placed in the fuel tank in the center of the UAV gravity center $\left(L_{\text {Center Tank }}=0.72 m\right)$. The fuel tank is located in the center of gravity since decreasing fuel does not change under conditions of airplane equilibrium.

Since the fuel tank location has been determined. In the second step, the size and volume of the tank should be calculated. Therefore, the volume and dimensions of the fuel tank are [25]:

$$
\begin{gathered}
\dot{m} f=P_{\text {max }} \times p s . f . c_{\text {max }} \rightarrow \dot{m} f=5 \times 10^{-4 \mathrm{~kg} / \mathrm{s}} \\
\dot{m} f=1.5 \times \dot{m} f \rightarrow \dot{m} f_{\text {max }}=7 . \dot{6} 5 \times 10^{-4 \mathrm{~kg} / \mathrm{s}}
\end{gathered}
$$

Due to the fuel used in UAVs with a density of about $\rho_{\text {Fuel }}=700^{\mathrm{kg} / \mathrm{m}^{3}}$, so the volume of the tank is [25]:

$$
\begin{gathered}
V_{\text {Tank }}=\frac{W_{\text {fuel }}}{\rho_{\text {fuel }}}=\frac{2.55}{600} \\
V_{\text {Tank }}=0.01245 \mathrm{~m}^{3}
\end{gathered}
$$

Therefore, if the cylinder tank is considered, the following numbers are obtained [25]

$$
\begin{aligned}
& \text { Tank Diameter }=0.15^{m} \\
& \text { Tank Length }=0.18^{m}
\end{aligned}
$$

Since the fuel tank is close to the UAV engine, a small pump is also used to reduce the weight of the UAV. Also, due to the ease of installation, maintenance, and maintenance of the UAV as much as possible, the velocity on top of the body is fitted with the dimensions of the tank, so that the tank can be pulled out of the body and poured fuel into it. For this purpose, two valves should be built on the tank and fuel holder must be provided for separating the tank from the fuel lines. Also for tank fueling, without its displacement, the two valves mounted above the tank are removed from the surface of the structure due to the structural frames and are properly insulated.

\subsection{Structural Tips}

Given that the UAV is designed to be identified, the UAV will carry out limited maneuvers. By analyzing the usual maneuvers and taking into account standards such as the fastest turnaround and the lowest radius of turnaround, distance, maneuver speed, and load analysis, the maximum load factor of 1.5 is selected to design the structure; therefore, structural analysis in order to make optimal design based on it.

In this UAV, the wing is made up of an interior section between the tail and the two outer parts connected to the interior. The overall structure of the wing is a foam core reinforced with a long spar being coated by the shell.

Due to the fact that a large portion of the wing has a flap, in order to strengthen the structure and counteract the torque created by the flap, a spar is also considered for the flap, which connects to the main wing. To strengthen the parts of the wings, which are affected by maximum stresses, as well as the reduction of the bow of the wing from the ribs in the desired locations, including the location of the canvas to the wings and the location of the wing to the body, as well as the sides of the flaps located.

What's so obvious is the usual form of body design to use frames that are connected by Longrun and are responsible for bearing the transverse and longitudinal loads applied to the body. In particular, the location of the motor connected to the body, which results in tiredness and ultimately failure of the structure, is due to vibrations.

It should also be noted that the largest structural investment in a UAV designed according to its shape should be made at the junction of the wing to the body where the landing gear and the place of application of all loads applied through the wing and tail and the place of transfer of this loading to another frame of body.

Also, given that the UAV is connected with two flat plates, an aluminum plate, composite materials or honeycomb instruments can be used to provide strength. However, the design of the canvas is less complicated, and the only remarkable thing about its design is the ability to withstand tensions and local considerations for conducting connection wires to the receiver. The above is also true of the connecting wings to the body.

To find a direction for UAV, we use a linear search method in the pixels of the background, which can be a time consuming and an obstacle to executing the program at runtime. Therefore, to increase the system performance and fix this problem, we will run a preprocessing stage and network image, so that in each section it is not possible to place more than one UAV. So in this way, if we find a UAV in a cell, we will no longer need to search for another UAV in that cell. In addition, the UAV can be easily tracked according to the previous frame.

\subsection{Camera and Antenna Location}

Because the purpose of the mission is on the ground, the camera is installed below the wing for a better view of the mission area. Two antennas, one above, for connection to the GPS system and one down for the Data Link installed. One is located above the body for easy installation and installation of systems inside the aircraft. The wings have the ability to detach from the body to facilitate transport and can be used as a gateway for installing systems that can be less accessible.

In the first step, accurate mapping of the camera can be accurately mapped to the plane. Since our goal is to use the system in the external environment, we have to choose a calibration method that is easy to execute and repeat without requiring special tools. In this method, in less than one minute, it is possible to map the fixed camera to the real position of that point in space by selecting only three 
points of the coordinate image of each point.

Also, using this method, you can display the areas under the care of multiple fixed cameras with a portable camera with an appropriate magnification. In Fig. 4, a demonstration of the position of the object and parameters related to its position is shown to the camera. Based on these parameters, we map the position of the object in the image to the required angles for moving the camera with high magnitude and horizontal-vertical orientation.

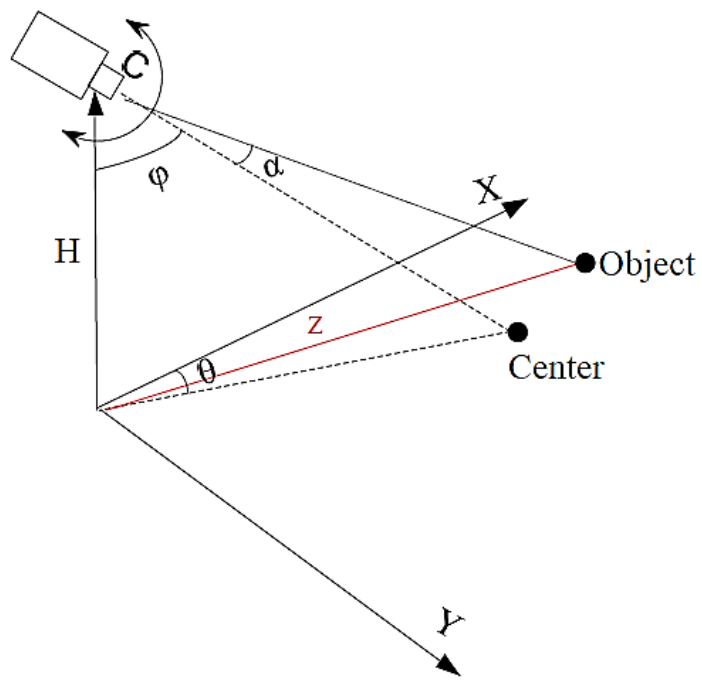

Figure 4. Related parameters of the camera, with the ability to zoom on a specific object

\section{Results}

In this paper, the design of the UAV was described in the light of the technology available in the country, and by comparing the results of the design with similar foreign samples and by designing a test sample, the design accuracy was somewhat determined. A summary of the design data is presented in Table 5.
Table 5. Designed quantities

\begin{tabular}{|c|c|}
\hline Total weight & 15.8 \\
\hline Empty weight & 12.14 \\
\hline Useful load weight & 2.01 \\
\hline Fuel weight & 2.50 \\
\hline Body length & 1.15 \\
\hline Body diameter & 0.2 \\
\hline Flap type & Simple \\
\hline Flap span & 3.6 \\
\hline Flap chord & 0.05 \\
\hline Maximum deformation angle & $60^{\circ}$ \\
\hline Location of flap & Trailing edge \\
\hline Aspect ratio & 16.3 \\
\hline Wing area & 0.5 \\
\hline Wing span & 3.6 \\
\hline Wing chord & 0.5 \\
\hline Wing rollback angle & $0^{\circ}$ \\
\hline Wings diledral angle & $0^{\circ}$ \\
\hline Installation angle of wing to body & $3^{\circ}$ \\
\hline Wing torsion angle & $0^{\circ}$ \\
\hline Distance of the landing gear of nose from tip & 0.15 \\
\hline Distance of the terminal landing gear of the tip & 0.45 \\
\hline $\begin{array}{l}\text { Transverse distance of back landing gear from } \\
\text { each other }\end{array}$ & 0.6 \\
\hline Height of landing gears & 0.22 \\
\hline
\end{tabular}

\subsection{Analysis of Designed Structure in ABAQUS Software}

This analysis was carried out statically and after entering the data about the wings and making the analysis model, the outcomes were extracted. The results of the shell failure analysis designed by the software and using the failure analysis are shown in Fig. 5. As shown in Fig. 5, all values obtained in the fracture analysis are acceptable and, as a result, the shell is designed to withstand the slip strength due to the pressure load due to the lift force. 


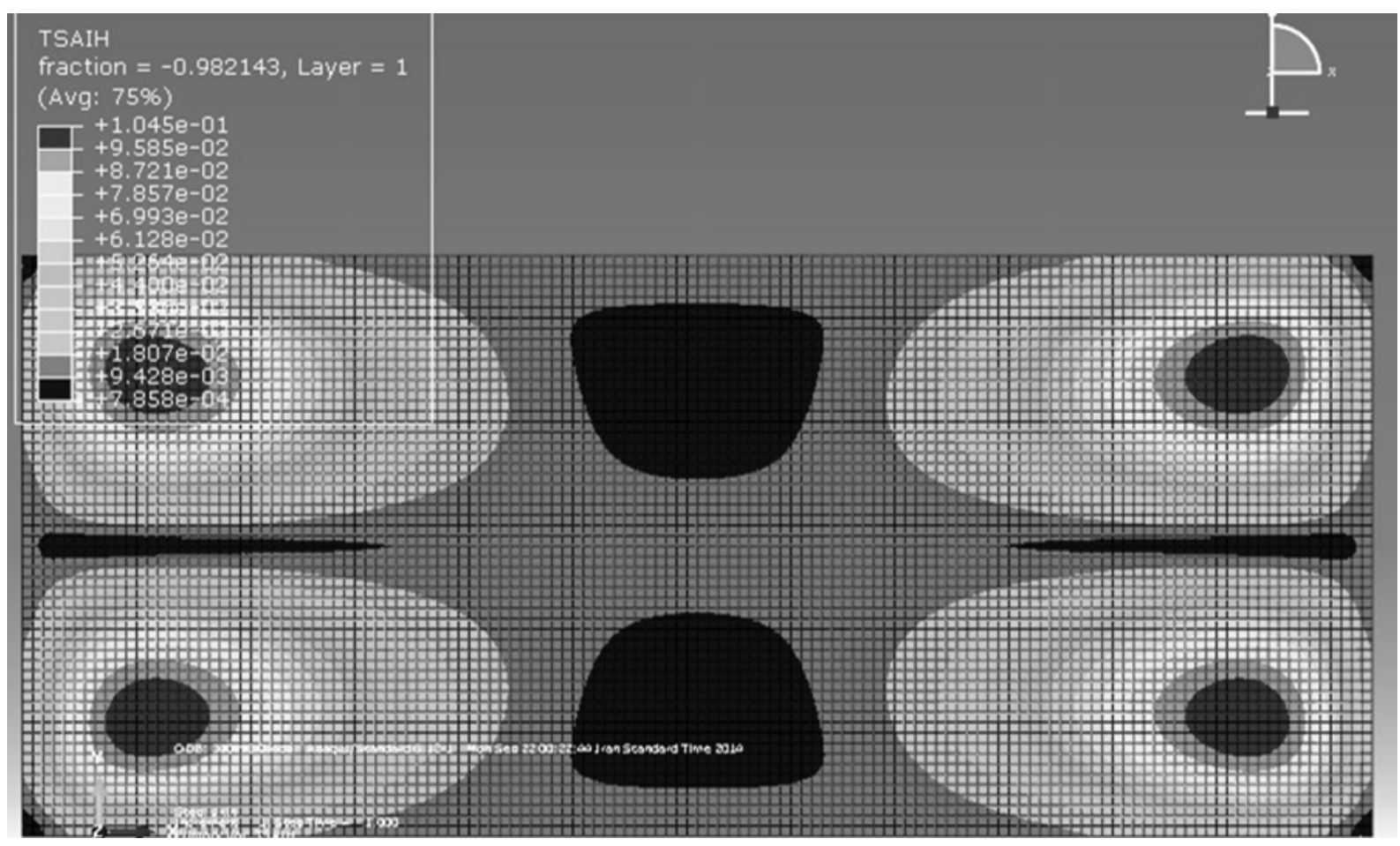

Figure 5. Analysis of failure direction for wing shell

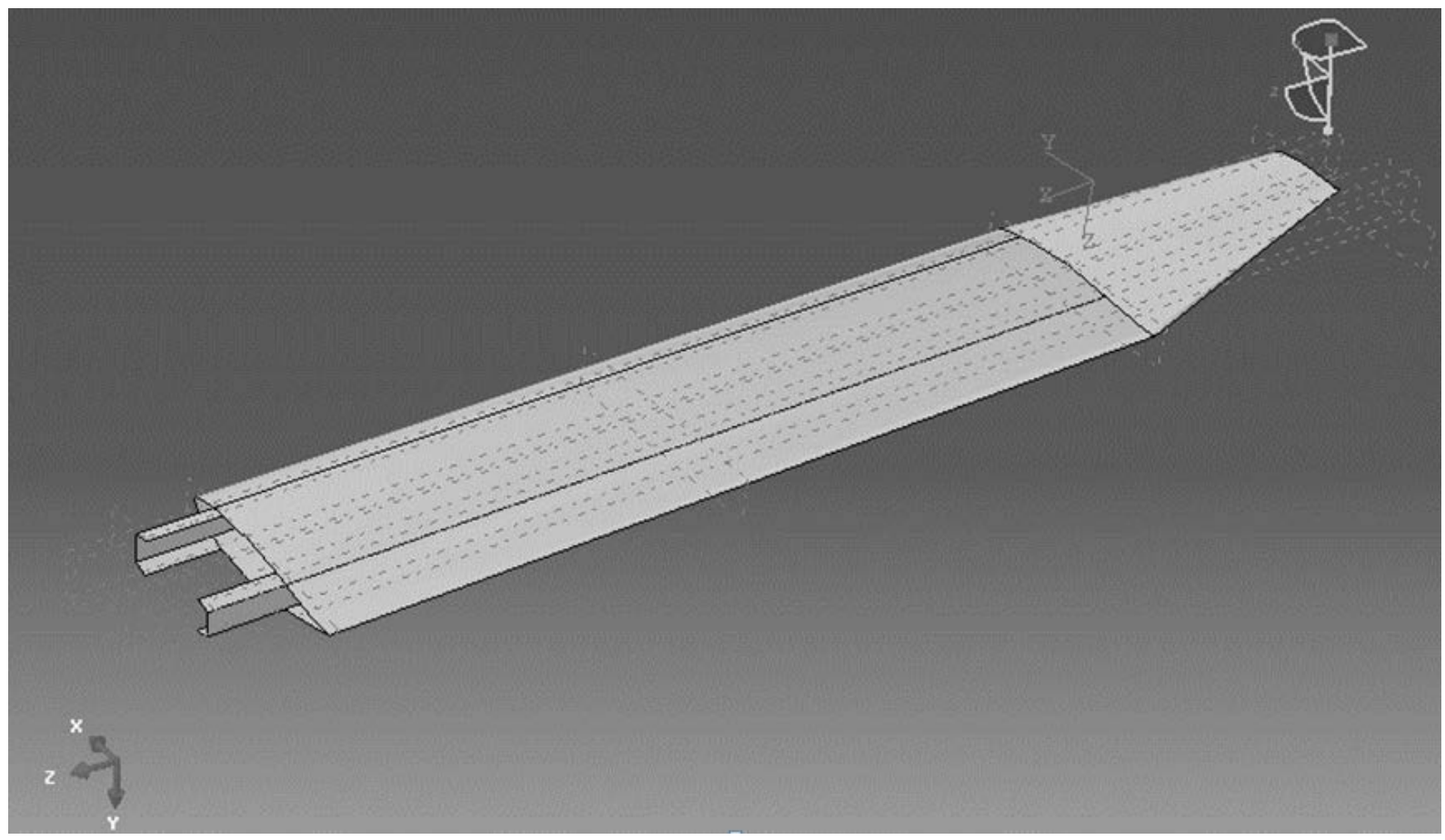

Figure 6. Preparing the model in the ABAQUS software

In Fig. 6, the model presented in the software is displayed. The preparation of a precise model in the software is one of the most important stages in the analysis process, and the final results of the analysis are highly dependent on the accuracy of the operation at this step. According to the results of the analysis for the entire structure of the wing and the stated explanation need to strengthen the wing structure in the shells and in the area of the shell to the front and back spar After strengthening of the mentioned sites based on the repetition of the calculations performed on the wings shell, without any other changes in the software sections, force analysis on the structure was performed and the results of the failure study are presented in Fig. 7. 


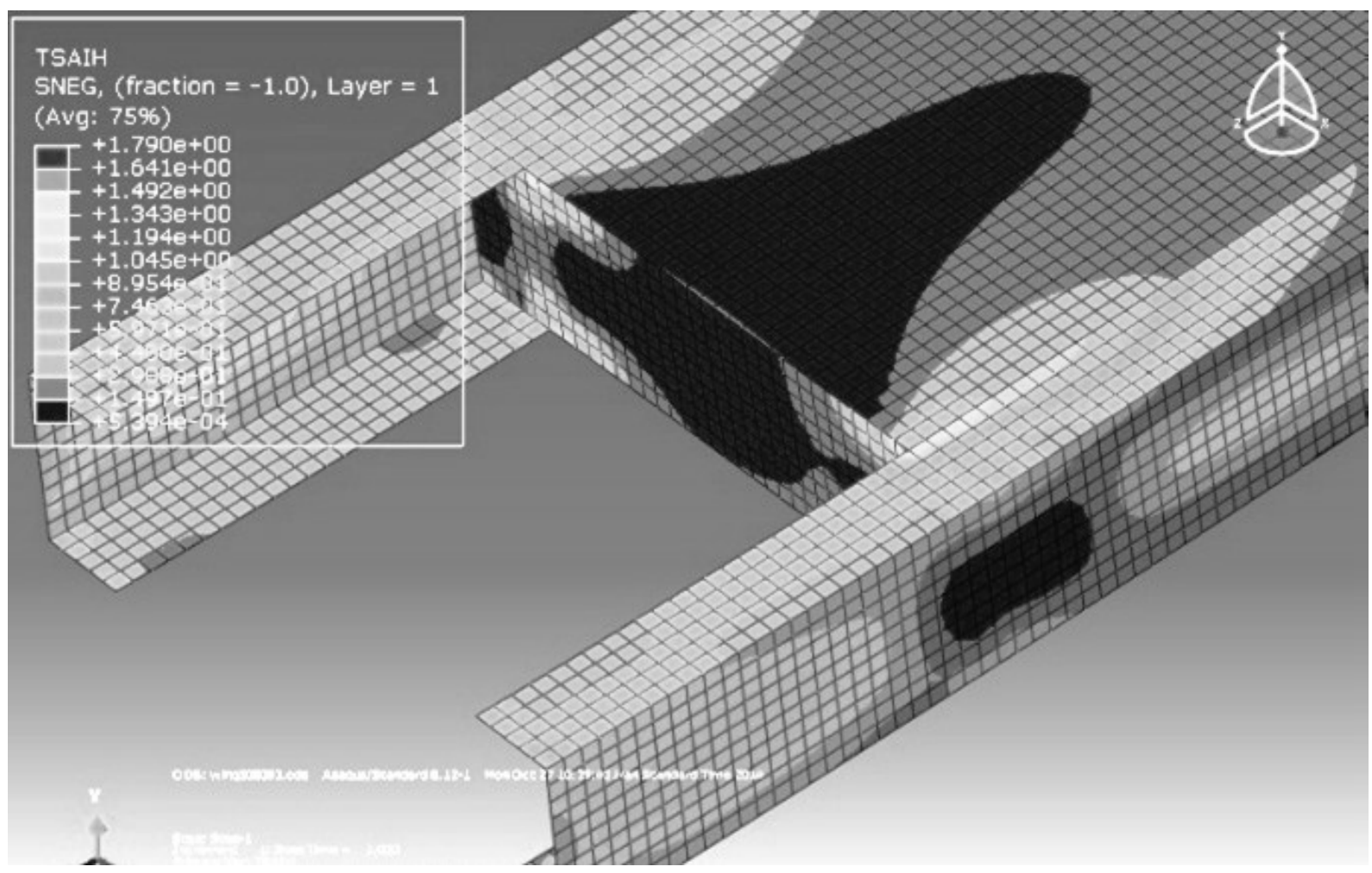

Figure 7. Lower spar wing in analysis of failure after reinforcement and increasing shell strength

The results show that with the reinforcement of the wings shell, failure in this section is not observed, however, spar itself was more stressed in very small areas than previous analyzes, and it was observed at the point of attachment of the spar to the layers of failure shell which, due to the proximity of the number of the analysis to the acceptable value in the fracture analysis, ignores it. In a general summary of analyzes and results, the strength of the wing structure versus the forces was confirmed.

\section{Conclusions}

In this study, we design and analysis UAV used for shooting and filming. Let us again mentioned the most important achievements and innovations in this study. According to the aerodynamic analysis, the loads can be determined for structural analysis, and accordingly based on the required structure of the UAV designed or reviewed. In this way, the tail torque also greatly reduces the need for the angle to neutralize the torque. The engine is designed to balance the torque and thus increase propulsion efficiency and reduce fuel consumption. The results show that the analysis has the appropriate accuracy and, on the other hand, the analysis of the level of failure of the layers in the software indicates the strength of the layers and the layout against the load on the wings in the most critical flight conditions. On the other hand, in areas of the designed wing structure, there was a need for reinforcement to be strengthened, which, according to the analysis carried out in these areas, was identified and the necessary amendments were applied. As a result, it can be said that the designed structure needs to be optimized and it can be deduced from the results of the analysis in regions where lower stresses are introduced into the structure, reducing the size of the layers and adding to more sensitive areas such as the root of the wings. On the other hand, in designing aerial structures, one of the most important factors is the weight loss of the structure. In this research, this is the designer's desired criterion, but it is necessary to consider the more coherent weight loss in optimizing the structure.

\section{REFERENCES}

[1] Esakki, B., Ganesan, S., Mathiyazhagan, S., Ramasubramanian, K., Gnanasekaran, B., Son, B., Park, S. W. ... Choi, J. S. (2018). Design of Amphibious Vehicle for Unmanned Mission in Water Quality Monitoring Using Internet of Things. Sensors (Basel, Switzerland), 18(10), 3318. doi:10.3390/s18103318

[2] F. Delgado, J. Carvalho, T. Coelho, and A. Dos Santos, "An Optical Fiber Sensor and Its Application in UAVs for Current Measurements,” Sensors, vol. 16, no. 11, p. 1800, 2016.

[3] Yang L.J., Esakki B., Chandrasekhar U., Hung K.C., Cheng C.M (2015). Practical flapping mechanisms for 20 cm-span micro air vehicles. Int. J. Micro Air Veh. 2015; 7: 
181-202. doi: 10.1260/1756-8293.7.2.181.

[4] Udayagiri C., Kulkarni M., Esakki B., Pakiriswamy S., Yang L.J (2016). Experimental Studies on 3D Printed Parts for Rapid Prototyping of Micro Aerial Vehicles. J. Appl. Sci. Eng. 2016;19:17-22

[5] Chandrasekhar U., Yang L.J., Esakki B., Suryanarayanan S., Salunkhe S (2017). Rapid prototyping of flapping mechanisms for monoplane and biplane ornithopter configurations. Int. J. Mod. Manuf. Technol. 2017;9:18-22

[6] Victor V. Klemas (2015). Coastal and Environmental Remote Sensing from Unmanned Aerial Vehicles: An Overview. Journal of Coastal Research, 31(5): 1260-1267.

[7] Anderson K, Gaston KJ (2013). Lightweight unmanned aerial vehicles will revolutionize spatial ecology. Frontiers in Ecology and the Environment. 2013;11:138-146

[8] Chabot D, Bird DM (2015). Wildlife research and management methods in the 21st century: where do unmanned aircraft fit in? Journal of Unmanned Vehicle Systems. 2015;3:137-155

[9] Goebel ME, Perryman WL, Hinke JT, Krause DJ, Hann NA, Gardner S, LeRoi DJ(2015). A small unmanned aerial system for estimating abundance and size of Antarctic predators. Polar Biology. 2015; 38:619-630

[10] Muzzamil, Ahmad Fadlilah \& Rosid, Nurhayyan \& Hanif, M \& Fadel, Nashwa \& Moelyadi, Mochammad \& Budiyono, Agus. (2018). Design and Development of Tube-Launched Unmanned Aerial Vehicle.

[11] Sun, Jingxuan \& Li, Boyang \& Jiang, Yifan \& Wen, Chih-yung. (2016). A Camera-Based Target Detection and Positioning UAV System for Search and Rescue (SAR) Purposes. Sensors. 16. 1778. 10.3390/s16111778.

[12] Muhammad Akmal Zulkipli and Khairul Nizam Tahar, "Multirotor UAV-Based Photogrammetric Mapping for Road Design,” International Journal of Optics, vol. 2018, Article ID 1871058, 7 pages, 2018. https://doi.org/10.1155/2018/1871058.

[13] M. A. Núñez, F. Buill, and M. Edo, “3D model of the Can Sadurní cave,” Journal of Archaeological Science, vol. 40, no. 12, pp. 4420-4428, 2013. View at Publisher - View at Google Scholar

[14] S. G. Kontogiannis and J. A. Ekaterinaris, "Design, performance evaluation and optimization of a UAV," Aerospace Science and Technology, vol. 29, no. 1, pp. 339-350, 2013. View at Publisher - View at Google Scholar · View at Scopus

[15] Terletzky P, Ramsey RD (2014). A semi-automated single day image differencing technique to identify animals in aerial imagery. PLoS ONE. 2014; 9: e1831 doi: 10.1371/journal.pone.0085239.

[16] Vermeulen C, Lejeune P, Lisein J, Sawadogo P, Bouché P (2013). Unmanned aerial survey of elephants. PLoS ONE. 2013; 8: e1831 doi: 10.1371/journal.pone.0054700.

[17] Yayla M., Sarsilmaz S.B., Mutlu T., Cosgun V., Kurtulus B., Kurtulus D.F., Tekinalp O. Dynamic Stability Flight Tests of Remote Sensing Measurement Capable Amphibious Unmanned Aerial Vehicle (A-UAV); Proceedings of the 7th Ankara International Aerospace Conference AIAC;
Ankara, Türkiye.

[18] Chomphunut Sutheerakul, Nopadon Kronprasert, Manop Kaewmoracharoen, Preda Pichayapan (2017). Application of Unmanned Aerial Vehicles to Pedestrian Traffic Monitoring and Management for Shopping Streets. Transportation Research Procedia. Volume 25, 2017, Pages 1717-1734

[19] McEvoy, J. F., Hall, G. P., \& McDonald, P. G. (2016). Evaluation of unmanned aerial vehicle shape, flight path and camera type for waterfowl surveys: disturbance effects and species recognition. PeerJ, 4, e1831. doi:10.7717/peerj.1831

[20] Ohood Al Nuaimi, Omar Almelhi, Abdulrahman Almarzooqi, Abdullah Al Saadi Al Mansoori, Slim Sayadi, Issacniwas Swamidoss (2018). Persistent surveillance with small Unmanned Aerial Vehicles (sUAV): a feasibility study. Proceedings Volume 10796, Electro-Optical Remote Sensing XII; 107960K

(2018) https://doi.org/10.1117/12.2326693

[21] Franco P. L. Franco; Denis S. Loubach ; André R. Fioravanti (2017). An embedded system design for a two-axis camera platform control used in unmanned aerial vehicles. IEEE. Latin American Robotics Symposium (LARS) and 2017 Brazilian Symposium on Robotics (SBR).

[22] Torres-Sánchez, J., López-Granados, F., De Castro, A. I., \& Peña-Barragán, J. M. (2013). Configuration and specifications of an Unmanned Aerial Vehicle (UAV) for early site specific weed management. PloS one, 8(3), e58210.

[23] Houghton\& Carruthers, "Aerodynamics for engineering", third edition, 1984.

[24] Janes Unmanned Aerial Vehichels \& Targets, 2003

[25] Mattingly \& Heiser \& Daley, "Constraint Analysis for electric powered Mini Aerial Vehicle”, Aircraft Engine 